Bull. Korean Math. Soc. 47 (2010), No. 3, pp. 623-632

DOI 10.4134/BKMS.2010.47.3.623

\title{
ON A POSITIVE SUBHARMONIC BERGMAN FUNCTION
}

\author{
Jung OK Kim And ERn Gun Kwon
}

Abstract. A holomorphic function $F$ defined on the unit disc belongs to $\mathcal{A}^{p, \alpha}(0<p<\infty, 1<\alpha<\infty)$ if

$$
\int_{U}|F(z)|^{p} \frac{1}{1-|z|}\left(1+\log \frac{1}{1-|z|}\right)^{-\alpha} d x d y<\infty .
$$

For boundedness of the composition operator defined by $C_{f} g=g \circ f$ mapping Blochs into $\mathcal{A}^{p, \alpha}$, the following (1) is a sufficient condition while (2) is a necessary condition.

$$
\begin{aligned}
& \text { (1) } \int_{0}^{1} \frac{1}{1-r}\left(1+\log \frac{1}{1-r}\right)^{-\alpha} M_{p}(r, \lambda \circ f)^{p} d r<\infty, \\
& \text { (2) } \int_{0}^{1} \frac{1}{1-r}\left(1+\log \frac{1}{1-r}\right)^{-\alpha+p}(1-r)^{p} M_{p}\left(r, f^{\sharp}\right)^{p} d r<\infty .
\end{aligned}
$$

\section{Introduction}

We introduce few facts that we need in the sequel, most of which are well known.

Let $U$ denote the open unit disc of the complex plane.

For $1<\alpha<\infty$ and $0<p<\infty$, let $\mathcal{A}^{p, \alpha}$ denote the weighted Bergman space of holomorphic functions on $U$, consisting of those holomorphic $f$ in $U$ for which

$$
\|f\|_{\mathcal{A}^{p, \alpha}}:=\left(\int_{U}|f(z)|^{p} \frac{1}{1-|z|}\left(1+\log \frac{1}{1-|z|}\right)^{-\alpha} d x d y\right)^{1 / p}<\infty .
$$

Note that $\mathcal{A}^{p, \alpha}$ is different from $A^{p, \alpha}$, the well-known weighted Bergman space of order $\alpha, \alpha>-1$, consisting of holomorphic functions $f$ in $U$ for which

$$
\|f\|_{A^{p, \alpha}}:=\left(\int_{U}|f(z)|^{p}(1-|z|)^{\alpha} d x d y\right)^{1 / p}<\infty .
$$

Received January 2, 2009.

2000 Mathematics Subject Classification. 32A37.

Key words and phrases. composition operator, Bloch space, weighted Bergman space.

This work was supported by KRF-2005-070-C00007. 
For functions defined in $U$ and for $0<p<\infty, 0 \leq r<1, M_{p}(r, f)$ is defined as usual by

$$
M_{p}(r, f)=\left(\frac{1}{2 \pi} \int_{0}^{2 \pi}\left|f\left(r e^{i \theta}\right)\right|^{p} d \theta\right)^{1 / p} .
$$

For $f$ with $|f|$ subharmonic in $U$, we set

$$
\|f\|_{p}:=\sup _{r} M_{p}(r, f) .
$$

Then the classical Hardy space $H^{p}=H^{p}(U)$ is the space of those $f$ holomorphic in $U$ for which $\|f\|_{p}<\infty$. The Yamashita [8] hyperbolic Hardy class $H_{\sigma}^{p}$ is defined as the set of those holomorphic self-maps $f$ of $U$ for which $\|\sigma(f)\|_{p}<\infty$, where $\sigma(z)$ denotes the hyperbolic distance of $z$ and 0 in $U$, i.e.,

$$
\sigma(z)=\frac{1}{2} \log \frac{1+|z|}{1-|z|}
$$

We set, following Yamashita,

$$
\lambda(f)=\log \frac{1}{1-|f|^{2}} \quad \text { and } \quad f^{\sharp}=\frac{\left|f^{\prime}\right|}{1-|f|^{2}}
$$

for holomorphic self-maps $f$ of $U$. It obvious that $f \in H_{\sigma}^{p}$ if and only if $\|\lambda(f)\|_{p}<\infty$ and that $f^{\sharp}$ is $\mathcal{M}$-invariant in the sense that $f^{\sharp}=(\varphi \circ f)^{\sharp}$ for any $\varphi \in \mathcal{M}$, where $\mathcal{M}$ is the group of all automorphisms of $U$.

The Bloch space $\mathcal{B}$ consists of holomorphic functions $h$ in $U$ for which

$$
\sup _{z \in U}\left|h^{\prime}(z)\right|\left(1-|z|^{2}\right)<\infty .
$$

This is a Banach space, if the norm $\|h\|_{\mathcal{B}}$ of $h \in \mathcal{B}$ is defined to be the sum of $|h(0)|$ and the left side of above inequality. A pair of Bloch functions $h_{j}, j=1,2$ are constructed such that

$$
\left(1-|z|^{2}\right)\left(\left|h_{1}^{\prime}(z)\right|+\left|h_{2}^{\prime}(z)\right|\right) \geq 1, \quad z \in U
$$

([6]). Then it follows that

$$
\frac{1}{1-|f|^{2}} \leq\left|h_{1}^{\prime} \circ f\right|+\left|h_{2}^{\prime} \circ f\right| \leq \frac{C}{1-|f|^{2}}
$$

for holomorphic self-maps $f$, where $C=2 \max \left\{\left\|h_{1}\right\|_{\mathcal{B}},\left\|h_{2}\right\|_{\mathcal{B}}\right\}$. For $h \in \mathcal{B}$, it follows from Schwarz-Pick's Lemma ([2]) that

$$
\left|(h \circ f)^{\prime}(z)\right| \leq\|h\|_{\mathcal{B}} f^{\sharp}(z) \leq\|h\|_{\mathcal{B}} \frac{1}{1-|z|^{2}}, \quad z \in U .
$$

For $f: U \rightarrow U$ be holomorphic, the composition operator $C_{f}$ generated by $f$ is defined by $C_{f} h=h \circ f, h \in \mathcal{B}$. Also, we define $C_{f}^{0}$ by $C_{f}^{0} h=h \circ f-h(0)$. See [7].

Our results in this note are as follows. 
Theorem 1. $\lambda(z)=\log \frac{1}{1-|z|^{2}},|z|<1$. Let $f: U \rightarrow U$ be holomorphic and $1<\alpha<\infty, 1 \leq p<\infty$. Then the following (1) implies (3) with $\delta=\alpha$; (3) implies (2) with $\delta=\alpha-p$, and (2) implies (1).

$$
\begin{gathered}
\int_{0}^{1} \frac{1}{1-r}\left(1+\log \frac{1}{1-r}\right)^{-\alpha} M_{p}(r, \lambda \circ f)^{p} d r<\infty . \\
\int_{0}^{1} \frac{1}{1-r}\left(1+\log \frac{1}{1-r}\right)^{-\delta}(1-r)^{p} M_{p}\left(r, f^{\sharp}\right)^{p} d r<\infty .
\end{gathered}
$$

After introducing simple but useful lemmas in Section 2, we will prove our main results in Section 3.

\section{Lemmas}

Lemma 1. Let $f: U \rightarrow U$ be holomorphic and $h: U \rightarrow C$ be holomorphic. Then, for $1 \leq p<\infty$ and $1<\alpha<\infty$,

$$
\begin{aligned}
& \int_{U} \frac{1}{1-|z|}\left(1+\log \frac{1}{1-|z|}\right)^{-\alpha}(1-|z|)^{p}\left|(h \circ f)^{\prime}(z)\right|^{p} d x d y \\
\leq & C_{\alpha, p} \int_{U} \frac{1}{1-|z|}\left(1+\log \frac{1}{1-|z|}\right)^{-\alpha}|(h \circ f)(z)-h(0)|^{p} d x d y,
\end{aligned}
$$

where $C_{\alpha, p}$ is a constant depending on $\alpha$ and $p$.

Proof. We show that there is a constant $C_{\alpha, p}$ depending only on $p$ and $\alpha$ such that

$$
\begin{aligned}
& \int_{0}^{1} \frac{1}{1-r}\left(1+\log \frac{1}{1-r}\right)^{-\alpha}(1-r)^{p} r d r \int_{0}^{2 \pi}\left|(h \circ f)^{\prime}\left(r e^{i \theta}\right)\right|^{p} d \theta \\
\leq & C_{\alpha, p} \int_{0}^{1} \frac{1}{1-r}\left(1+\log \frac{1}{1-r}\right)^{-\alpha} r d r \int_{0}^{2 \pi}\left|(h \circ f)\left(r e^{i \theta}\right)-h(0)\right|^{p} d \theta .
\end{aligned}
$$

Let $h \circ f-h(0)=F$. Then

$$
\int_{T}\left|(h \circ f)^{\prime}\left(r e^{i \theta}\right)\right|^{p} \frac{d \theta}{2 \pi}=M_{p}\left(r, F^{\prime}\right)^{p} .
$$

By $[1$, p. 80$]$

$$
M_{p}\left(r, F^{\prime}\right) \leq \frac{M_{p}\left(\frac{1+r}{2}, F\right)}{\left(\frac{1+r}{2}\right)^{2}-r^{2}}=\frac{M_{p}\left(\frac{1+r}{2}, F\right)}{\left(\frac{1+3 r}{2}\right)\left(\frac{1-r}{2}\right)} \leq 4 \frac{M_{p}\left(\frac{1+r}{2}, F\right)}{1-r}
$$


whence

$$
\begin{aligned}
& \int_{0}^{1} \frac{1}{1-r}\left(1+\log \frac{1}{1-r}\right)^{-\alpha}(1-r)^{p} M_{p}\left(r, F^{\prime}\right)^{p} r d r \\
\leq & 4^{p} \int_{0}^{1} \frac{1}{1-r}\left(1+\log \frac{1}{1-r}\right)^{-\alpha}(1-r)^{p}\left(\frac{M_{p}\left(\frac{1+r}{2}, F\right)}{1-r}\right)^{p} r d r \\
\leq & 4^{p} \int_{0}^{1} \frac{1}{1-r}\left(1+\log \frac{1}{1-r}\right)^{-\alpha} M_{p}\left(\frac{1+r}{2}, F\right)^{p} r d r \\
\leq & C_{\alpha, p} \int_{0}^{1} \frac{1}{1-r}\left(1+\log \frac{1}{1-r}\right)^{-\alpha} M_{p}(r, F)^{p} r d r,
\end{aligned}
$$

where $C_{\alpha, p}$ is a constant depending on $\alpha$ and $p$. Therefore

$$
\begin{aligned}
& \int_{0}^{1} \frac{1}{1-r}\left(1+\log \frac{1}{1-r}\right)^{-\alpha}(1-r)^{p} r d r \int_{0}^{2 \pi}\left|(h \circ f)^{\prime}\left(r e^{i \theta}\right)\right|^{p} d \theta \\
\leq & C_{\alpha, p} \int_{0}^{1} \frac{1}{1-r}\left(1+\log \frac{1}{1-r}\right)^{-\alpha} r d r \int_{0}^{2 \pi}\left|(h \circ f)\left(r e^{i \theta}\right)-h(0)\right|^{p} d \theta .
\end{aligned}
$$

Lemma 2. Let $f: U \rightarrow U$ be holomorphic. Then

$$
\frac{\partial}{\partial r}\left(\log \frac{1}{1-|f|^{2}}\right) \leq 2 f^{\sharp} .
$$

Proof. It is easy to see that

$$
\frac{\partial}{\partial r}\left(\log \frac{1}{1-|f|^{2}}\right)=\frac{\partial}{\partial r}(-\log (1-f \bar{f}))=\frac{f_{r} \bar{f}+f \bar{f}_{r}}{1-f \bar{f}},
$$

where $f_{r}=\frac{\partial f}{\partial r}$. But since

$$
f_{r}=\frac{\partial f}{\partial r}=\frac{\partial f}{\partial z} \frac{\partial z}{\partial r}=f^{\prime} e^{i \theta}
$$

and

it follows that

$$
\bar{f}_{r}=\frac{\partial \bar{f}}{\partial r}=\frac{\partial \bar{f}}{\partial \bar{z}} \frac{\partial \bar{z}}{\partial r}=\bar{f}^{\prime} e^{-i \theta}
$$

$$
\begin{aligned}
\frac{\partial}{\partial r}\left(\log \frac{1}{1-|f|^{2}}\right) & =\frac{\bar{f} f^{\prime} e^{i \theta}+\bar{f}^{\prime} e^{-i \theta} f}{1-|f|^{2}} \\
& =\frac{2 R e\left(\bar{f} f^{\prime} e^{i \theta}\right)}{1-|f|^{2}} \\
& \leq \frac{2\left|f^{\prime}\right||f|}{1-|f|^{2}}
\end{aligned}
$$

Noting that $|f| \leq 1$, we obtain

$$
\frac{\partial}{\partial r}\left(\log \frac{1}{1-|f|^{2}}\right) \leq 2 f^{\sharp} .
$$


Lemma 3. Let $\lambda(z)=\log \frac{1}{1-|z|^{2}},|z|<1$. Let $f: U \rightarrow U$ be holomorphic and $1<a<\infty, 1<b<\infty, 1 \leq p<\infty$. Then

$$
\begin{aligned}
& \int_{0}^{1} \frac{1}{1-r}\left(1+\log \frac{1}{1-r}\right)^{-b} M_{p}(r, \lambda \circ f)^{a} d r \\
\leq & \frac{a}{b-1}|\lambda \circ f(0)|^{a} \\
& +\left(\frac{2 a}{b-1}\right)^{a} \int_{0}^{1} \frac{1}{1-r}\left(1+\log \frac{1}{1-r}\right)^{-b+a}(1-r)^{a} M_{p}\left(r, f^{\sharp}\right)^{a} d r .
\end{aligned}
$$

Proof. Integrating by parts, we have

$$
\begin{aligned}
& \int_{0}^{1} \frac{1}{1-r}\left(1+\log \frac{1}{1-r}\right)^{-b} M_{p}(r, \lambda \circ f)^{a} d r \\
= & \left.\frac{1}{-b+1}\left(1+\log \frac{1}{1-r}\right)^{-b+1} M_{p}(r, \lambda \circ f)^{a}\right|_{0} ^{1} \\
& -\int_{0}^{1} \frac{1}{-b+1}\left(1+\log \frac{1}{1-r}\right)^{-b+1} \frac{\partial}{\partial r} M_{p}(r, \lambda \circ f)^{a} d r \\
= & \frac{-1}{-b+1} M_{p}(0, \lambda \circ f)^{a}-\int_{0}^{1} \frac{1}{-b+1}\left(1+\log \frac{1}{1-r}\right)^{-b+1} \frac{\partial}{\partial r} M_{p}(r, \lambda \circ f)^{a} d r \\
= & \frac{1}{b-1}|\lambda \circ f(0)|^{a}+\frac{1}{b-1} \int_{0}^{1}\left(1+\log \frac{1}{1-r}\right)^{1-b} \frac{\partial}{\partial r} M_{p}(r, \lambda \circ f)^{a} d r,
\end{aligned}
$$

so that

$$
\begin{aligned}
& \int_{0}^{1} \frac{1}{1-r}\left(1+\log \frac{1}{1-r}\right)^{-b} M_{p}(r, \lambda \circ f)^{a} d r \\
= & \frac{1}{b-1}|\lambda \circ f(0)|^{a}+\frac{1}{b-1} \int_{0}^{1}\left(1+\log \frac{1}{1-r}\right)^{1-b} \frac{\partial}{\partial r} M_{p}(r, \lambda \circ f)^{a} d r .
\end{aligned}
$$

But, since

$$
\begin{aligned}
\frac{\partial}{\partial r} M_{p}(r, \lambda \circ f)^{a} & =a M_{p}(r, \lambda \circ f)^{a-1} \frac{\partial}{\partial r} M_{p}(r, \lambda \circ f) \\
& =\frac{a}{p} M_{p}(r, \lambda \circ f)^{a-p} p M_{p}(r, \lambda \circ f)^{p-1} \frac{\partial}{\partial r} M_{p}(r, \lambda \circ f) \\
& =\frac{a}{p} M_{p}(r, \lambda \circ f)^{a-p} \frac{\partial}{\partial r} M_{p}(r, \lambda \circ f)^{p}
\end{aligned}
$$

we have

$$
\frac{\partial}{\partial r} M_{p}(r, \lambda \circ f)^{a}=\frac{a}{p} M_{p}(r, \lambda \circ f)^{a-p} \frac{\partial}{\partial r} M_{p}(r, \lambda \circ f)^{p} .
$$


And since

$$
\begin{aligned}
\frac{\partial}{\partial r}\left|\lambda \circ f\left(r e^{i \theta}\right)\right|^{p} \leq\left|\frac{\partial}{\partial r}\left(\lambda \circ f\left(r e^{i \theta}\right)\right)^{p}\right| & =\left|p\left(\lambda \circ f\left(r e^{i \theta}\right)\right)^{p-1} \frac{\partial}{\partial r}\left(\lambda \circ f\left(r e^{i \theta}\right)\right)\right| \\
& \leq\left|p\left(\lambda \circ f\left(r e^{i \theta}\right)\right)^{p-1}\right|\left|\frac{\partial}{\partial r}\left(\lambda \circ f\left(r e^{i \theta}\right)\right)\right| \\
& \leq 2 p\left|\left(\lambda \circ f\left(r e^{i \theta}\right)\right)^{p-1}\right| f^{\sharp}\left(r e^{i \theta}\right),
\end{aligned}
$$

where we used Lemma 2 in last inequality, we have

$$
\left.\left.\left|\frac{\partial}{\partial r}\right| \lambda \circ f\left(r e^{i \theta}\right)\right|^{p}|\leq 2 p| \lambda \circ f\left(r e^{i \theta}\right)\right|^{p-1} f^{\sharp}\left(r e^{i \theta}\right) .
$$

By (2.2) and (2.3),

$$
\begin{aligned}
\left|\frac{\partial}{\partial r} M_{p}(r, \lambda \circ f)^{a}\right| & =\left|\frac{a}{p} M_{p}(r, \lambda \circ f)^{a-p} \frac{\partial}{\partial r} M_{p}(r, \lambda \circ f)^{p}\right| \\
& \leq\left.\frac{a}{p} M_{p}(r, \lambda \circ f)^{a-p} \int_{0}^{2 \pi}\left|\frac{\partial}{\partial r}\right| \lambda \circ f\left(r e^{i \theta}\right)\right|^{p} \mid \frac{d \theta}{2 \pi} \\
& \leq \frac{a}{p} M_{p}(r, \lambda \circ f)^{a-p} \int_{0}^{2 \pi} 2 p\left|\lambda \circ f\left(r e^{i \theta}\right)\right|^{p-1} f^{\sharp}\left(r e^{i \theta}\right) \frac{d \theta}{2 \pi}
\end{aligned}
$$

Thus it follows from Hölder's inequality that

$$
\begin{aligned}
& \left|\frac{\partial}{\partial r} M_{p}(r, \lambda \circ f)^{a}\right| \\
\leq & \frac{a}{p} M_{p}(r, \lambda \circ f)^{a-p} \int_{0}^{2 \pi} 2 p\left|\lambda \circ f\left(r e^{i \theta}\right)\right|^{p-1} f^{\sharp}\left(r e^{i \theta}\right) \frac{d \theta}{2 \pi} \\
\leq & 2 a M_{p}(r, \lambda \circ f)^{a-p}\left(\int_{0}^{2 \pi}\left(\left|\lambda \circ f\left(r e^{i \theta}\right)\right|^{p-1}\right)^{p /(p-1)} \frac{d \theta}{2 \pi}\right)^{(p-1) / p} \\
& \times\left(\int_{0}^{2 \pi}\left|f^{\sharp}\left(r e^{i \theta}\right)\right|^{p} \frac{d \theta}{2 \pi}\right)^{1 / p} \\
= & 2 a M_{p}(r, \lambda \circ f)^{a-p} M_{p}(r, \lambda \circ f)^{p-1} M_{p}\left(r, f^{\sharp}\right) \\
= & 2 a M_{p}(r, \lambda \circ f)^{a-1} M_{p}\left(r, f^{\sharp}\right)
\end{aligned}
$$

for $p>1$. This is also true when $p=1$. Consequently, the last integral of (2.1) is dominated by

$$
\begin{aligned}
& 2 a \int_{0}^{1}\left(1+\log \frac{1}{1-r}\right)^{1-b} M_{p}(r, \lambda \circ f)^{a-1} M_{p}\left(r, f^{\sharp}\right) d r \\
\leq & 2 a\left\{\int_{0}^{1} \frac{1}{1-r}\left(1+\log \frac{1}{1-r}\right)^{-b} M_{p}(r, \lambda \circ f)^{a} d r\right\}^{1-1 / a} \\
& \times\left\{\int_{0}^{1}\left(\frac{1}{1-r}\right)^{1-a}\left(1+\log \frac{1}{1-r}\right)^{-b+a} M_{p}\left(r, f^{\sharp}\right)^{a} d r\right\}^{1 / a},
\end{aligned}
$$


where Hölder's inequality has been used again. Therefore by (2.4), (2.1) is dominated by

$$
\begin{aligned}
& \int_{0}^{1} \frac{1}{1-r}\left(1+\log \frac{1}{1-r}\right)^{-b} M_{p}(r, \lambda \circ f)^{a} d r \\
= & \frac{1}{b-1}|\lambda \circ f(0)|^{a}+\frac{1}{b-1} \int_{0}^{1}\left(1+\log \frac{1}{1-r}\right)^{1-b} \frac{\partial}{\partial r} M_{p}(r, \lambda \circ f)^{a} d r \\
\leq & \frac{1}{b-1}|\lambda \circ f(0)|^{a} \\
& +\frac{2 a}{b-1}\left\{\int_{0}^{1} \frac{1}{1-r}\left(1+\log \frac{1}{1-r}\right)^{-b} M_{p}(r, \lambda \circ f)^{a} d r\right\}^{1-1 / a} \\
& \times\left\{\int_{0}^{1}\left(\frac{1}{1-r}\right)^{1-a}\left(1+\log \frac{1}{1-r}\right)^{-b+a} M_{p}\left(r, f^{\sharp}\right)^{a} d r\right\}^{1 / a} .
\end{aligned}
$$

In order to obtain the desired inequality, let

$$
A=\int_{0}^{1} \frac{1}{1-r}\left(1+\log \frac{1}{1-r}\right)^{-b} M_{p}(r, \lambda \circ f)^{a} d r
$$

and

$$
B=\int_{0}^{1} \frac{1}{1-r}\left(1+\log \frac{1}{1-r}\right)^{-b+a}(1-r)^{a} M_{p}\left(r, f^{\sharp}\right)^{a} d r
$$

for a moment. Then (2.5) can be expressed as

$$
A \leq \frac{1}{b-1}|\lambda \circ f(0)|^{a}+A^{1-1 / a} \times\left[\left(\frac{2 a}{b-1}\right)^{a} B\right]^{1 / a} .
$$

Using

$$
A^{\alpha} B^{1-\alpha} \leq \alpha A+(1-\alpha) B \quad(0 \leq \alpha \leq 1),
$$

we have

equivalently

$$
A \leq \frac{1}{b-1}|\lambda \circ f(0)|^{a}+\left[\left(1-\frac{1}{a}\right) A+\left(\frac{2 a}{b-1}\right)^{a} \frac{1}{a} B\right],
$$

Therefore

$$
\frac{1}{a} A \leq \frac{1}{b-1}|\lambda \circ f(0)|^{a}+\left(\frac{2 a}{b-1}\right)^{a} \frac{1}{a} B
$$

$$
A \leq \frac{a}{b-1}|\lambda \circ f(0)|^{a}+\left(\frac{2 a}{b-1}\right)^{a} B
$$

That is,

$$
\begin{aligned}
& \int_{0}^{1} \frac{1}{1-r}\left(1+\log \frac{1}{1-r}\right)^{-b} M_{p}(r, \lambda \circ f)^{a} d r \\
\leq & \frac{a}{b-1}|\lambda \circ f(0)|^{a} \\
& +\left(\frac{2 a}{b-1}\right)^{a} \int_{0}^{1} \frac{1}{1-r}\left(1+\log \frac{1}{1-r}\right)^{-b+a}(1-r)^{a} M_{p}\left(r, f^{\sharp}\right)^{a} d r .
\end{aligned}
$$


This completes the proof.

Lemma 4. Let $g \in \mathcal{B}$ and $f \in H^{\infty}(U)$ with $|f|<1$. Then

$$
|g \circ f(z)-g(0)| \leq\|g\|_{\mathcal{B}} \sigma(f(z)), z \in U .
$$

Proof. For $w \in U$,

$$
\begin{aligned}
|g(w)-g(0)| & =\left|\int_{0}^{w} g^{\prime}(z) d z\right|=\left|\int_{0}^{|w|} g^{\prime}(t \zeta) \zeta d t\right| \\
& \leq \int_{0}^{|w|}\left|g^{\prime}(t \zeta)\right| d t \\
& \leq \int_{0}^{|w|}\|g\|_{\mathcal{B}} \frac{1}{1-|t \zeta|^{2}} d t=\|g\|_{\mathcal{B}} \int_{0}^{|w|} \frac{d t}{1-t^{2}} \\
& =\|g\|_{\mathcal{B}} \frac{1}{2} \log \frac{1+|w|}{1-|w|}=\|g\|_{\mathcal{B}} \sigma(w),
\end{aligned}
$$

whence

$$
|g \circ f(z)-g(0)| \leq\|g\|_{\mathcal{B}} \sigma(f(z)), z \in U \text {. }
$$

\section{Proof of the results}

Proof of Theorem 1. Suppose (1) holds. Using Minkowski's inequality with those $h_{j}, j=1,2$ of (2.1) and Lemma 1, we obtain

$$
\begin{aligned}
& \left\{\int_{U} \frac{1}{1-|z|}\left(1+\log \frac{1}{1-|z|}\right)^{-\alpha}(1-|z|)^{p}\left(f^{\sharp}(z)\right)^{p} d x d y\right\}^{1 / p} \\
\leq & \left\{\int_{U} \frac{1}{1-|z|}\left(1+\log \frac{1}{1-|z|}\right)^{-\alpha}(1-|z|)^{p}\left(\sum_{j=1}^{2}\left|\left(h_{j} \circ f\right)^{\prime}(z)\right|\right)^{p} d x d y\right\}^{1 / p} \\
\leq & \sum_{j=1}^{2}\left\{\int_{U} \frac{1}{1-|z|}\left(1+\log \frac{1}{1-|z|}\right)^{-\alpha}(1-|z|)^{p}\left|\left(h_{j} \circ f\right)^{\prime}(z)\right|^{p} d x d y\right\}^{1 / p} \\
\leq & C_{\alpha, p} \sum_{j=1}^{2}\left\{\int_{U} \frac{1}{1-|z|}\left(1+\log \frac{1}{1-|z|}\right)^{-\alpha}\left|\left(h_{j} \circ f\right)(z)-h_{j}(0)\right|^{p} d x d y\right\}^{1 / p},
\end{aligned}
$$

where $C_{\alpha, p}$ is a constant depending on $\alpha$ and $p$.

Since

$$
\begin{aligned}
& \left\{\int_{U} \frac{1}{1-|z|}\left(1+\log \frac{1}{1-|z|}\right)^{-\alpha}\left|\left(h_{j} \circ f\right)(z)-h_{j}(0)\right|^{p} d x d y\right\}^{1 / p} \\
\leq & \left.\left\|h_{j}\right\|_{\mathcal{B}} \sup _{\substack{h \in \mathcal{B} \\
\|h\|_{\mathcal{B}} \leq 1}}\left\{\int_{U} \frac{1}{1-|z|}\left(1+\log \frac{1}{1-|z|}\right)^{-\alpha}|(h \circ f)(z)-h(0)|\right)^{p} d x d y\right\}^{1 / p},
\end{aligned}
$$


$j=1,2$, from $(3.1)$ we have

$$
\begin{aligned}
& \left\{\int_{U} \frac{1}{1-|z|}\left(1+\log \frac{1}{1-|z|}\right)^{-\alpha}(1-|z|)^{p}\left(f^{\sharp}(z)\right)^{p} d x d y\right\}^{1 / p} \\
\leq & C_{\alpha, p} \sup _{\substack{h \in \mathcal{B} \\
\|h\|_{\mathcal{B}} \leq 1}}\left\{\int_{U} \frac{1}{1-|z|}\left(1+\log \frac{1}{1-|z|}\right)^{-\alpha}|(h \circ f)(z)-h(0)|^{p} d x d y\right\}^{1 / p} \\
= & C_{\alpha, p} \sup _{\substack{\|h \in \mathcal{B}\\
\| h \|_{\mathcal{B}} \leq 1}}\|h \circ f-h(0)\|_{\mathcal{A}^{p, \alpha}} \\
= & C_{\alpha, p}\left\|C_{f}^{0}\right\| .
\end{aligned}
$$

Thus (3) holds.

Also, (3) with $\delta=\alpha-p \Longrightarrow(2)$ is proved in Lemma 3. And (2) $\Longrightarrow$ (1) follows directly from Lemma 4 with an application of Minkowski's inequality: For $g \in \mathcal{B}$,

$$
\begin{aligned}
& \int_{0}^{1} \frac{1}{1-r}\left(1+\log \frac{1}{1-r}\right)^{-\alpha} M_{p}(r, g \circ f)^{p} d r \\
\leq & \|g\|_{\mathcal{B}}^{p} \int_{0}^{1} \frac{1}{1-r}\left(1+\log \frac{1}{1-r}\right)^{-\alpha} M_{p}(r, 1+\sigma \circ f)^{p} d r<\infty .
\end{aligned}
$$

The proof is complete.

\section{References}

[1] P. L. Duren, Theory of $H^{p}$ spaces, Academic Press, New York, 1970.

[2] J. B. Garnett, Bounded Analytic Functions, Academic Press, New York, 1981.

[3] E. G. Kwon, Composition of Blochs with bounded analytic functions, Proc. Amer. Math. Soc. 124 (1996), no. 5, 1473-1480.

[4] (1999), no. 1, 97-103.

[5] E. G. Kwon and J. K. Lee, Norm of a composition operator on the Bloch space, Preprint.

[6] W. Ramey and D. Ullrich, Bounded mean oscillation of Bloch pull-backs, Math. Ann. 291 (1991), no. 4, 591-606.

[7] A. E. Taylor and D. C. Lay, Introduction to Functional Analysis, John Wiley \& Sons, New York-Chichester-Brisbane, 1980.

[8] S. Yamashita, Hyperbolic Hardy classes and hyperbolically Dirichlet-finite functions, Hokkaido Math. J. 10 (1981), Special Issue, 709-722.

JUNG OK KIM

Department of Mathematics Education

Andong National University

ANDONG 760-749, KoreA

E-mail address: loloki@hanmail.net 
ERn Gun Kwon

Department of Mathematics Education

Andong National University

ANDONG 760-749, KoreA

E-mail address: egkwon@andong.ac.kr 